ORIGINAL ARTICLE

\title{
Antibiotic Prescribing in Outpatient Before and After Implementation of the Urban Family Physician and Referral System
}

\author{
EHSAN NABOVATI ${ }^{1}$, HAMED MAHMOUDI ${ }^{2 *}$, REZA ABBASI ${ }^{3}$, SAEED BARZEGARI ${ }^{4}$, HOSSEIN AKBARI $^{5}$, MEHRDAD \\ FARZANDIPOUR ${ }^{6}$ \\ ${ }^{1}$ Department of Health Information Management \& Technology, School of Allied Health Professions, Kashan University of Medical Sciences, \\ Kashan, Iran, Email: enabovati@gmail.com, ORCID: 0000-0003-0430-4637 \\ ${ }^{2}$ Department of Health Information Management \& Technology, School of Allied Health Professions, Kashan University of Medical Sciences, \\ Kashan, Iran, Email: mahmoodi.hit95@gmail.com, ORCID:0000-0002-1669-2481 \\ ${ }^{3}$ Department of Health Information Management \& Technology, School of Allied Health Professions, Kashan University of Medical Sciences, \\ Kashan, Iran, Email: rezaabba30_2001@yahoo.com, ORCID:0000-0003-3648-963X \\ ${ }^{4}$ Faculty Member at University of Medical Sciences, Mazandaran, Amol, Iran, Email: barz_saeed@yahoo.com, ORCID: 0000-0003-0393- \\ 1436 \\ ${ }^{5} \mathrm{PhD}$ of Biostatistics, Kashan University of Medical Science (KAUMS), Email: akbari1350_h@yahoo.com, ORCID: 0000-0002-3002-9205 \\ ${ }^{6}$ Department of Health Information Management \& Technology, School of Allied Health Professions, Kashan University of Medical Sciences, \\ Kashan, Iran, Email: farzandipour m@kaums.ac.ir, ORCID: 0000-0001-9738-7771 \\ ${ }^{*}$ Corresponding author: Hamed Mahmoudi, Email: mahmoodi.hit95@gmail.com
}

\begin{abstract}
Background

The Family Physician and Referral System was piloted in Mazandaran and Fars provinces, Iran in 2012. The goal of this program was to improve the provision of health services including medication prescription.

Objective

This study aimed to determine the trends in antibiotic prescribing in outpatient before and after implementation of the Family Physician and Referral System in Babol, Iran from 2010 to 2018.

Methods

In this retrospective study, all prescriptions of urban family physicians in Babol, which were registered in the database of the Health Services Insurance Organization of Mazandaran province, were included. The trends of average number of items per prescription, percentage of antibiotics per prescription, frequency of antibiotic groups, and cost of antibiotics were calculated using SQL Server and IBM SPSS version 22.

Results

The average number of items per prescription was found to be $3.4 \pm 1.82$, and $2.8 \pm 1.60$, and percentage of antibiotics per prescription was $49 \%$ and $32 \%$ in 2010 and 2018 , respectively. The most prescribed antibiotic groups were penicillin (54.8\%) and cephalosporin (39.9\%). Amoxicillin 500mg capsules $(13.1 \%)$, metronidazole $250 \mathrm{mg}(10.2 \%)$, and ciprofloxacin $500 \mathrm{mg}(8.2 \%)$ were the most prescribed antibiotics. Also, the mean cost of prescriptions containing antibiotics compared to the total prescriptions had decreased from $22 \%$ in 2010 to $5.5 \%$ in 2018.

Conclusion

The pattern of medication prescription especially antibiotics, improved after the implementation of the Family Physician and Referral System. Due to the positive impact of the Family Physician and Referral System on medication prescription by physicians, we recommend the implementation of this program in low and middleincome countries.
\end{abstract}

Keywords: Antibiotic, medication prescribing, Health system reform plan, Family physician, Trend

\section{INTRODUCTION}

Antibiotics are one of the most effective and widely used groups of drugs for treatment of diseases. Despite the benefits and widespread use, their inappropriate and irrational prescription causes antibiotic resistance, increase duration of treatment and recovery, increase mortality, and finally, they impose various costs on the health system and society $(1,2)$. More than 235 million doses of antibiotics are consumed in the world every year (3). Germany spent about 772 million euros to produce antibiotics, however, more than half were used inappropriately and irrationally in 2012 (4). At least 25,000 and 70,000 deaths is reported each year due to antibiotic resistance in Europe and the United States, according to the Centers for Disease Control and Prevention (CDC) (5-8). Antibiotic resistance was responsible for at least 3.2 million extra hospitalization days and 38481 deaths in Thailand (9).
According to the statistics, antibiotics are the most prescribed and used drugs in Iran (10). The total annual expenditure of antibiotics in Iran is between 1200 to 1800 billion Rials and the consumption of antibiotics is equal almost to the total consumption in Europe $(3,11)$. The previous studies in Iran have demonstrated that $51-59 \%$ of medications prescribed by general practitioners are antibiotics $(3,12-15)$. Improving the pattern of prescribing and consuming drugs, especially antibiotics, as well as reducing the costs of their consumption, is one of the most important goals and tasks of health managers and policy makers (16). Given the effective performance of the Health System Reform Plan, in particular the Family Physician and Referral System, world health organization (WHO) considers this project as the global effort to improve the quality, effectiveness, equity, and cost reduction in the health care(17). Therefore, most of high and middle income 
countries, including China, Canada, Switzerland, Norway, the United Arab Emirates, and Saudi Arabia, have made reforms such as implementing the Health System Reform Plan and Family Physicians Program. Timely and consistent access to family physicians, reducing unnecessary referrals, improving rational use of drugs, as well as improving treatment methods and patient satisfaction are among the advantages of this reform (1823). The Ministry of Health and Medical Education (MoHME) in Iran implemented the urban Family Physician and Referral System as one of the major reform in the Iranian health system in $2012(24,25)$.

This program was implemented to improve disease prevention, diagnosis, treatment, improve quality of life, access to healthcare, and provide a model for rational prescription of drugs (26-28). Despite the important role of general practitioners in providing healthcare to patients, they received fewer resources and facilities compared to specialists. Therefore, the Family Physician and Referral System created strategic reforms in the Iranian health system(29, 30). In addition, the family physician program has developed a framework for medication prescription that has had a great impact on improving the rational prescribing and use of medications $(31,32)$. This reform was firstly piloted in Mazandaran and Fars, the north and south provinces in Iran with three and four million population in 2012.

The results of a study, which was conducted in the US during 2011-2015, showed that the prescribing antibiotics in outpatient and inappropriate prescribing of antibiotics decreased by $2.1 \%$ and $3.9 \%$, respectively after implementing a monitoring program(33). One of the best ways to determine the medication prescription pattern is to evaluate physicians' prescriptions. In our knowledge, this is the first study in antibiotic prescribing to examine the trends among outpatients before and after implementation of the urban Family Physician and Referral System in a middle income countries such as Iran (11, 15, 34, 35). Therefore, the aim of this study was to investigate the trends in antibiotic prescribing among outpatients before and after implementation of the urban Family Physician and Referral System in one of the largest cities of Mazandaran province, Iran.

\section{METHODS}

\section{Study area and population}

This is a retrospective study which was conducted in 2020 in Babol, Iran. All prescriptions of urban family physicians in Babol, which were registered in the database of the Health Services Insurance Organization of Mazandaran province, were included in the study. The prescription ID, patient insurance code, drugs generic code, number and cost of prescribed medications were extracted from the database. Antibiotics were classified into ten groups based on the American hospital formulary service (AHFS-Class)(36).

\section{Statistical analysis}

SQL Server was used to analyze the data after extraction from the database of the Health Services Insurance Organization. The trends of the average number of items per prescription, percentage of antibiotics per prescription, frequency of antibiotic groups, and cost of antibiotics were calculated by SQL Server 2019 Express Edition and IBM SPSS version 22.

\section{Results}

In total, 4,284,171 prescriptions and 13,625,842 medications prescribed by urban family physicians were investigated. The average number of items per prescription and percentage of antibiotics per prescription were found to be $3.1 \pm 1.74$ and $41.54 \% \pm 5.61$, respectively. The highest $(49 \%)$ and lowest $(32.07 \%)$ rates of antibiotic prescribing were reported in 2010 and 2018. The highest (3.4 numbers) and the lowest (2.8 numbers) average number of items per prescription were reported in to 2010 and 2018, respectively. The results showed that the percentage of antibiotics and the number of items per prescription has decreased from 2010-2018 (Figures 1, 2).

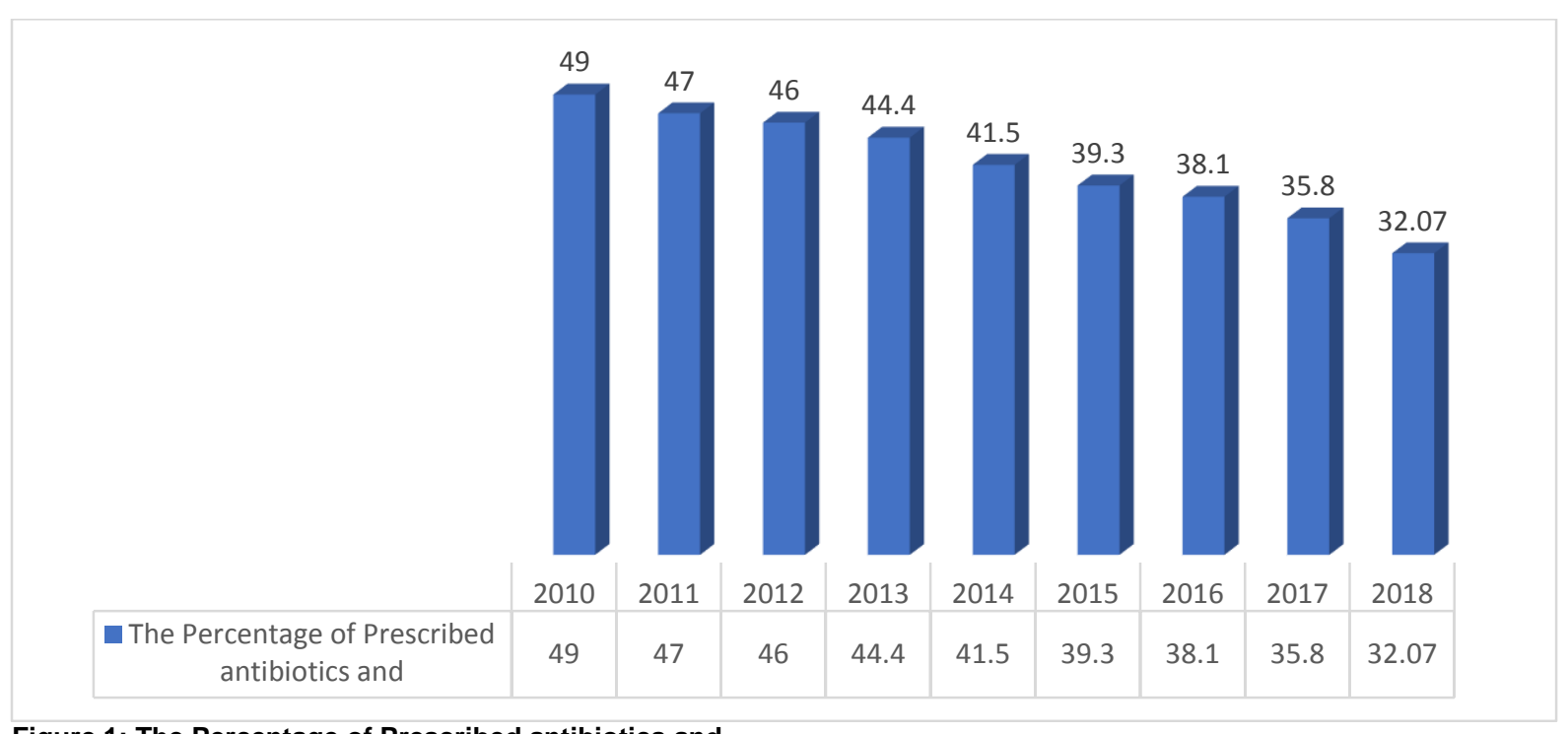

Figure 1: The Percentage of Prescribed antibiotics and 


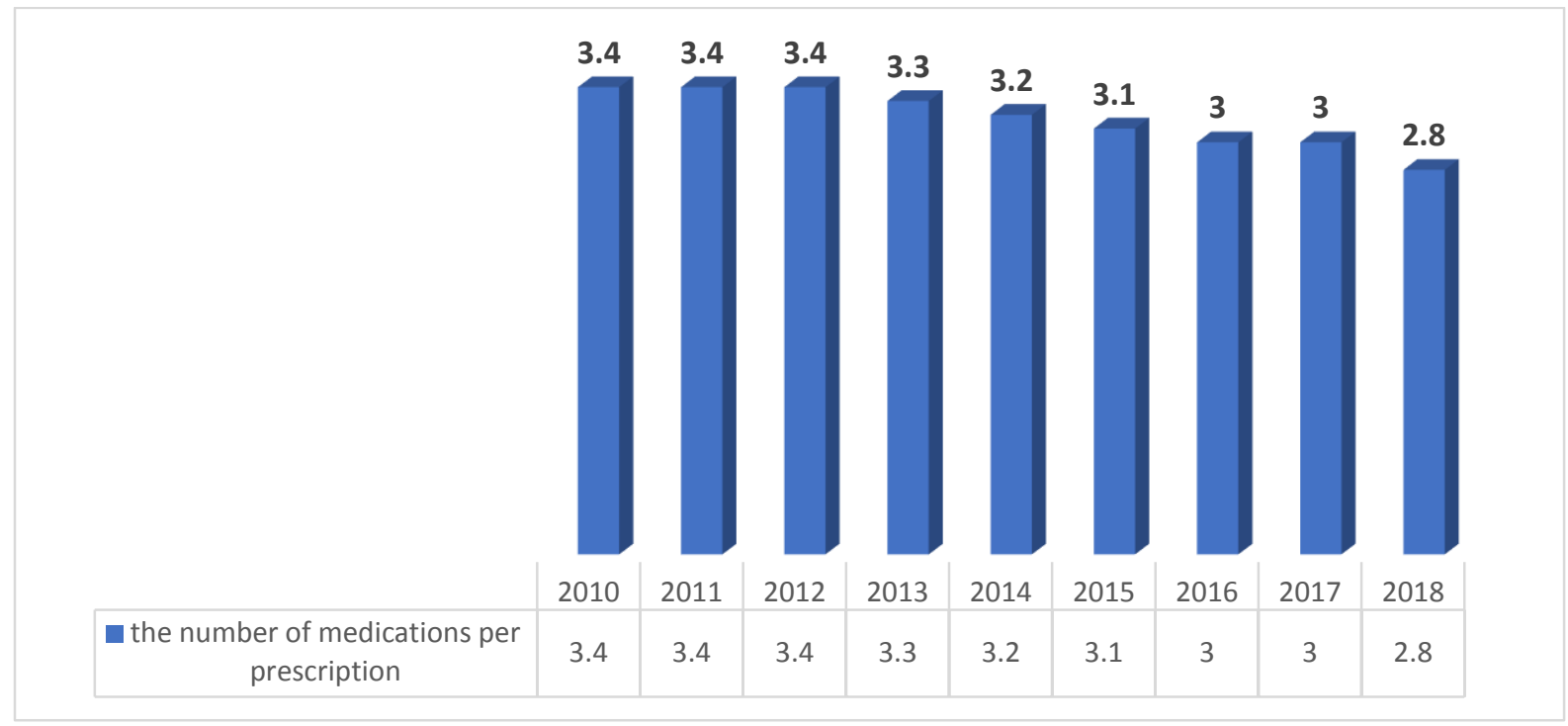

Figure 2: the number of medications per prescription

Penicillin were the most frequently prescribed classes of antibiotics (54.8\%) followed by cephalosporin (39.9\%), miscellaneous-antibiotics (15.17\%), quinolones (14\%), and aminoglycosides (12.17\%). Also, Amoxicillin 500-mg

capsules (13.1\%), metronidazole 250-mg (10.2\%), ciprofloxacin 500-mg (8.2\%), cefixime $400 \mathrm{mg}(9.03 \%)$, and Clidinium-c tablets $(7 \%)$ were the most frequently prescribed antibiotics (Figures 3, 4).

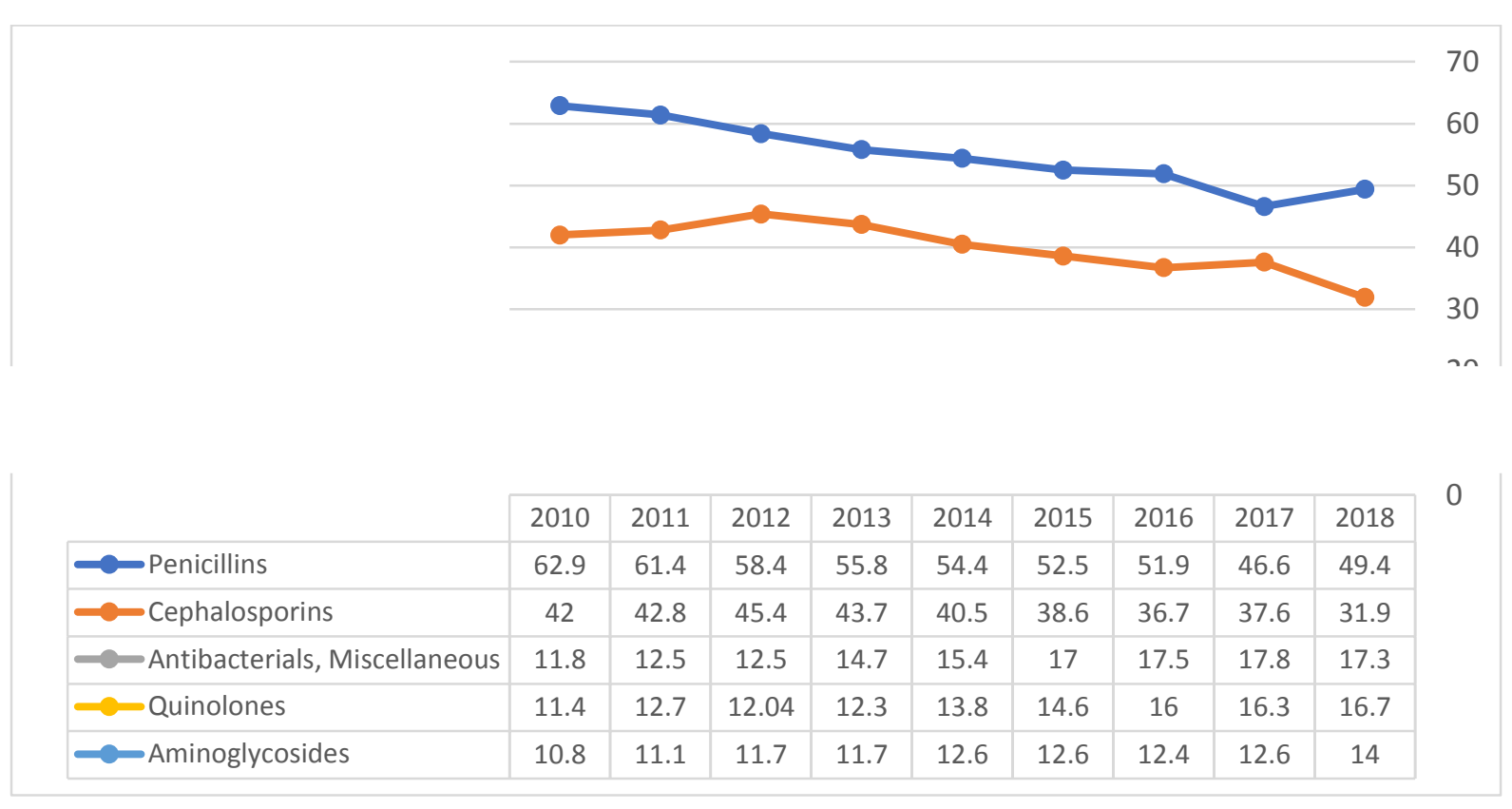

Figure 3: The most frequently prescribed antibiotic classes 


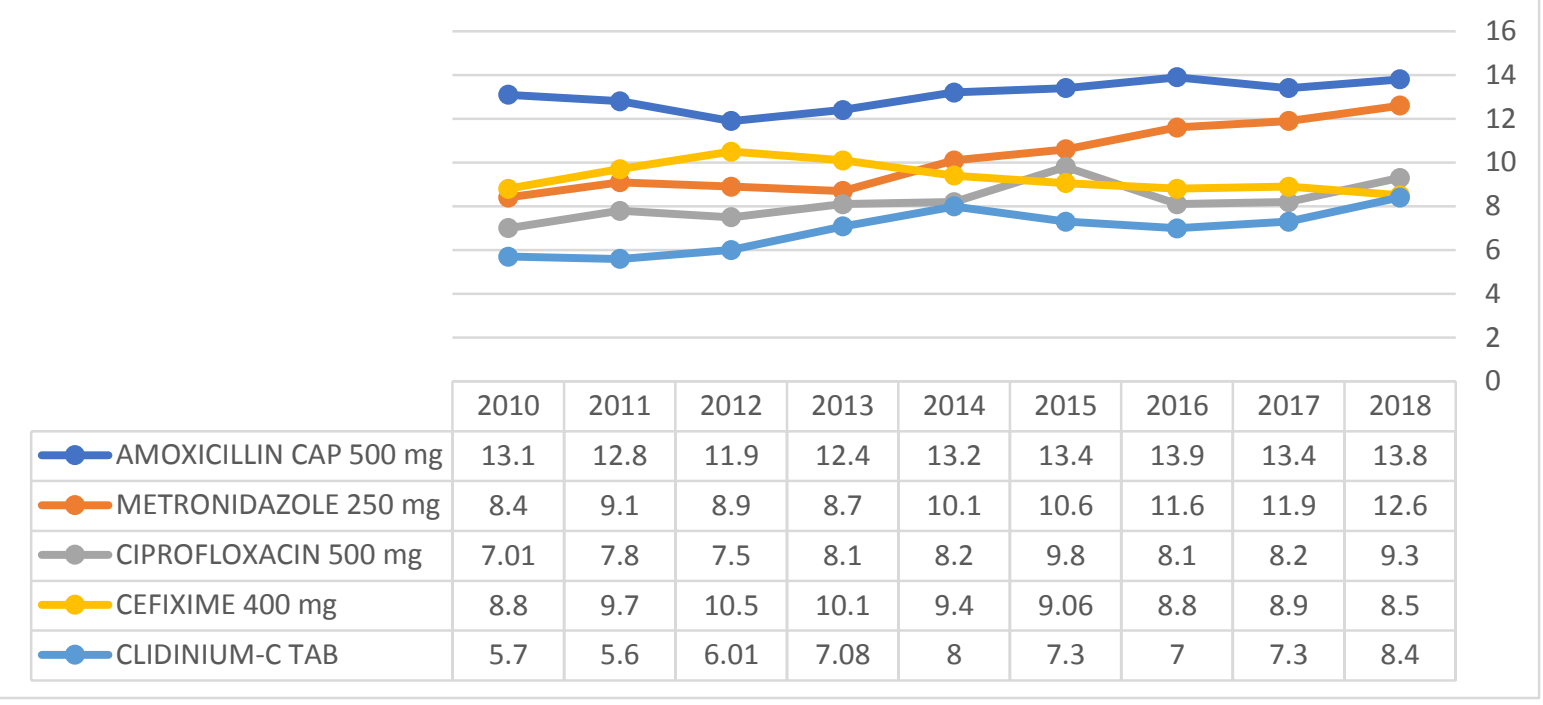

Figure 4: The most frequently prescribed antibiotics

Proportion of the total cost of prescribed antibiotics has decreased from 2010 (22\% of total costs of medications) to $2018(5.5 \%$ of total costs of medications). Furthermore, the lowest and highest cost of prescribed antibiotics were in $2011(8,616,913,691$ IRR) and $2016(19,485,536,932$ IRR), respectively (Figures 5,6 ).

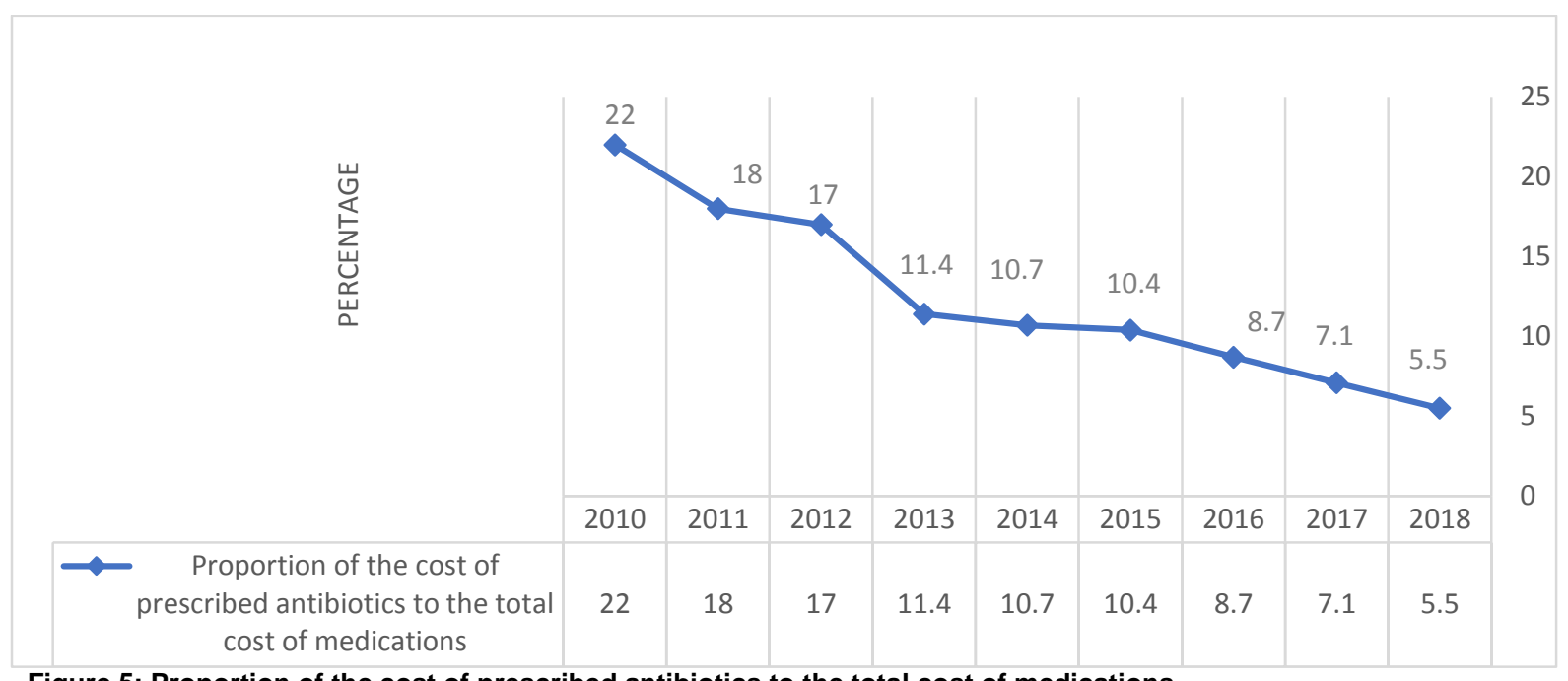

Figure 5: Proportion of the cost of prescribed antibiotics to the total cost of medications 


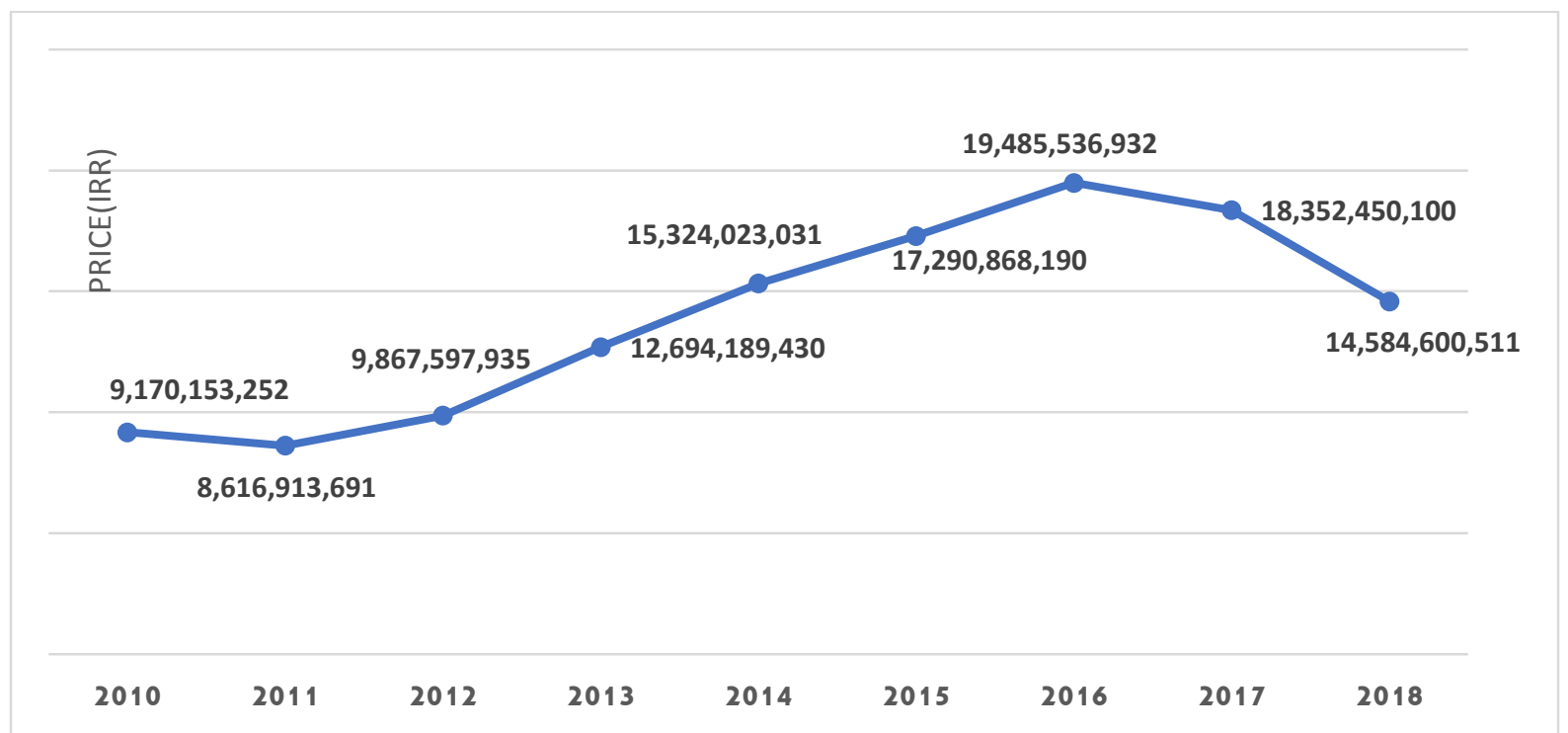

Figure 6: The price of prescribed antibiotics

\section{DISCUSSION}

In this study, more than four million urban family physicians' prescriptions have been investigated. The average number of items per prescription, percentage of antibiotics per prescription, frequency of antibiotic groups, and the proportion of the total cost of prescribed antibiotics have decreased after implementation of the urban Family Physician and Referral System. Penicillin, cephalosporin, miscellaneous antibiotics, quinolones, and aminoglycosides were the most frequently prescribed classes of antibiotics. Additionally, amoxicillin 500-mg capsules, metronidazole 250-mg, and ciprofloxacin 500-mg were the most frequently prescribed antibiotics.

We found that the average number of items per prescription was decreased by implementing Family Physician and Referral System. Similar to this study, Khadivi et al (37) (2014) retrospectively assessed the number of prescriptions per year before and after implementation of rural family physician program in health centers of Isfahan from 2004 to 2011. Their findings showed that the mean number of medications per prescription decreased from 4.27 to 4.11 following rural family physician program. According to the executive instruction of the family physician program, there are some restrictions on prescribing medications. Based on these restrictions, the average number of items per prescription by each family physician should not be more than 2.5 , otherwise, according to the instruction No. 02 of the Ministry of Health and Medical Education (MoHME) of Iran, they would be subject to deductions $(32,38)$. It seems that this restriction has been effective in reducing the average number of items per prescription. In contrast with our findings, the results of another study within the $\mathrm{WHO}$ African region showed that the average number of items per prescription increased from 2.2 in 2006 to 5.6 in 2015(39). The implementation of urban Family Physician and Referral System and improving of the policies and prescription in the Mazandaran province could be one possible reason of this discrepancy.
The mean of antibiotics percentage per prescription by urban family physicians was $41.5 \% \pm 5.61$, which is lower than overall mean of antibiotic prescribing in Iran (50\%)(3) and higher than recommended standard by WHO $(30 \%)(40)$. According to our results, implementation of the urban Family Physician and Referral System in Mazandaran province has decreased the mean of antibiotics percentage per prescription. The results of a study conducted in Kerman province showed that the trend in antibiotic prescribing decreased from 44\% in 2010 to $39 \%$ in $2015(41)$, which is consistent with our findings. The most important identified causes of antibiotic prescription reduction are continuing medical education and improving the knowledge of pharmacists, physicians, and patients about the irrational antibiotics prescribing. In contrary to our findings, Asenso Ofori(39), reported that the trend of antibiotic prescribing increased from 33.7\% in 1995 to $62.8 \%$ in 2015 in African countries. It seems that aging, epidemics, and the lack of effective monitoring and surveillance programs such as family physician program could increase antibiotics prescribing.

In our study, penicillin and cephalosporin were identified as the most widely prescribed groups of antibiotics. Consistent with these findings, the results of previous studies in Iran and other Asian and European countries showed that penicillin and cephalosporin are the most frequently prescribed antibiotic classes (30, 42, 43). A possible reason for high utilization of broad-spectrum antibiotics might be physicians' mindset, assuming that the broad-spectrum antibiotics are the best treatments to cure many infections. In fact, they rely on empirical treatment, instead of waiting for a laboratory test results(43).

Therefore, it is recommended that physicians prescribe antibiotics based on laboratory test results.

In our study, the most frequently prescribed antibiotics were amoxicillin 500-mg capsules, metronidazole $250-\mathrm{mg}$, ciprofloxacin 500-mg, and cefixime $400 \mathrm{mg}$, and clidinium-c tablets. The results of some studies in Iran showed that the amoxicillin, ciprofloxacin and cefixime were the most common prescribed antibiotics $(10,44)$. The highest rates of 
prescribed antibiotics are amoxicillin, ciprofloxacin and metronidazole in Cameroon and Ethiopia $(45,46)$. It seems that the main reason for prescribing these antibiotics is the inclusion in the class of broad-spectrum antibiotics.

According to our findings, the cost of prescribed antibiotics by family physicians increased from 2010 to 2015 . These findings are consistent with another study conducted in Kerman, Iran. Although the cost of antibiotics has been recently increased(41), proportion of the total cost of prescribed antibiotics shows a reduction from $22 \%$ in 2010 to $5.5 \%$ in 2018 . This is lower than the average cost $(28 \%)$ which reported in Iran(47).

The results of this study indicate that, despite rising antibiotics prices alongside with economic crises, the urban family physician program has been effective in reducing antibiotics prescription. The Ministry of Health and Medical Education (MoHME) of Iran has developed a framework for prescribing antibiotics, which has had an effective impact on reducing the prescription of antibiotics through a punitive mechanism $(32,38)$.

\section{Strengths and weaknesses}

This study would appear to be one of the first studies to investigate the effect of urban Family Physicians Program and Referral System on antibiotic prescribing in Iran. We reviewed more than four million prescriptions of urban family physicians. These prescriptions are related to the Iranian Health Services Insurance Organization, which is one of the two main insurance organizations in the country. Further studies are recommended to investigate the prescriptions in other insurance organizations before and after the Family Physician and Referral System implementation. The findings of our study may be beneficial to health ministry, insurance organizations, as well as health policymakers in different countries in order to promote the quality and quantity of medication prescription.

\section{Conclusion}

In this study, we found that the urban Family Physician and Referral System could be considered a crucial reform in the Iranian health system given the decreasing trend of the average number of items per prescription, percentage of antibiotics per prescription, frequency of antibiotic groups, and the proportion of the total cost of prescribed antibiotics. According to the results of the study, the antibiotic prescribing after implementation of the urban Family Physician and Referral System is lower than the overall mean of antibiotic prescribing in Iran and still higher than most European and even African countries. Due to the success of urban Family Physician and Referral System in reducing the medications prescribing, especially antibiotics, we suggest to implement this program in countries where family physician program have not yet been implemented.

Funding: There is no funding for this study.

Ethics approval and consent to participate: This study was approved by ethics committee of Kashan University of Medical Sciences (IR.KAUMS.NUHEPM.REC.1398.008).

Competing interests: The authors declare no conflict of interest.

\section{REFERENCES}

1. Gandra S, Barter D, Laxminarayan R. Economic burden of antibiotic resistance: how much do we really know? Clinical microbiology and infection. 2014;20(10):973-80.
2. Amaha ND, Weldemariam DG, Abdu N, Tesfamariam EH. Prescribing practices using $\mathrm{WHO}$ prescribing indicators and factors associated with antibiotic prescribing in six community pharmacies in Asmara, Eritrea: a cross-sectional study. Antimicrobial Resistance \& Infection Control. 2019;8(1):1-7.

3. Hosseinzadeh F, Sadeghieh Ahari S, Mohammadian-erdi A. Survey the antibiotics prescription by general practitioners for outpatients in Ardabil City in 2013. Journal of Ardabil University of Medical Sciences. 2016;16(2):140-50.

4. Zweigner J, Meyer E, Gastmeier P, Schwab F. Rate of antibiotic prescriptions in German outpatient care-are the guidelines followed or are they still exceeded? GMS hygiene and infection control. 2018;13.

5. Golkar Z, Bagasra O, Pace DG. Bacteriophage therapy: a potential solution for the antibiotic resistance crisis. The Journal of Infection in Developing Countries. 2014;8(02):129-36.

6. Bailey LC, Forrest CB, Zhang P, Richards TM, Livshits A, DeRusso PA. Association of antibiotics in infancy with early childhood obesity. JAMA pediatrics. 2014;168(11):1063-9.

7. Llor C, Bjerrum L. Antimicrobial resistance: risk associated with antibiotic overuse and initiatives to reduce the problem. Therapeutic advances in drug safety. 2014;5(6):229-41.

8. Didgar F, Sarmadian H, Ghasemikhah R. Antimicrobial resistance pattern of Gram-negative bacilli isolated of ValiAsr Hospital wards in Arak. ISMJ. 2014;17(5):938-47.

9. Sumpradit $\mathrm{N}$, Chongtrakul $\mathrm{P}$, Anuwong $\mathrm{K}$, Pumtong $\mathrm{S}$, Kongsomboon K, Butdeemee P, et al. Antibiotics Smart Use: a workable model for promoting the rational use of medicines in Thailand. Bulletin of the World Health Organization. 2012;90:905-13.

10. Nabovati E, TaherZadeh Z, Eslami S, Abu-Hanna A, Abbasi $R$. Antibiotic prescribing in inpatient and outpatient settings in Iran: a systematic review and meta-analysis study. Antimicrobial Resistance \& Infection Control. 2021;10(1):116.

11. Roumie CL, Halasa NB, Grijalva CG, Edwards KM, Zhu Y, Dittus RS, et al. Trends in antibiotic prescribing for adults in the United States-1995 to 2002. Journal of general internal medicine. 2005;20(8):697.

12. Karimi A, Haerizadeh M, Soleymani F, Haerizadeh M, Taheri F. Evaluation of medicine prescription pattern using World Health Organization prescribing indicators in Iran: A crosssectional study. Journal of research in pharmacy practice. 2014;3(2):39.

13. Afrasiabian S, Koolani A, Barari M, Hajibagheri K, Moradi G, Mohsenpour B. Evaluation of the status of antibiotic prescription in patients admitted to a teaching hospital in west of Iran. Chronic Diseases Journal. 2018;5(1):1-7.

14. Ahmadi F, Zarei E. Prescribing patterns of rural family physicians: a study in Kermanshah Province, Iran. BMC public health. 2017;17(1):908.

15. Zareshahi R, Haghdoost A, Asadipour A, Sadeghirad B. Rational usage of drug indices in the prescriptions of kerman medical practitioners in 2008. Journal of Rafsanjan University of Medical Sciences. 2012;11(6):523-36.

16. Bairami F, Soleymani F, Rashidian A. Improving injectable medicines prescription in outpatient services: a path towards rational use of medicines in Iran. International journal of health policy and management. 2016;5(5):321.

17. Rivo ML. Practicing in the new millennium: do you have what it takes? Family practice management. 2000;7(1):35.

18. Smith RV. Opus Optimus: A Model for Renewing Life's Later Years: Greenleaf Book Group; 2017.

19. Du J, Lu X, Wang Y, Cui S, Guo A, Coid D, et al. Mutual referral: a survey of GPs in Beijing. Family practice. 2012;29(4):441-7.

20. Pojskic N, Mackeigan L, Boon $\mathrm{H}$, Ellison $\mathrm{P}$, Breslin C. Ontario family physician readiness to collaborate with 
community pharmacists on drug therapy management. Research in social and administrative pharmacy. $2011 ; 7(1): 39-50$

21. de Bruin SR, Versnel N, Lemmens LC, Molema CC Schellevis FG, Nijpels G, et al. Comprehensive care programs for patients with multiple chronic conditions: a systematic literature review. Health policy. 2012;107(23):108-45.

22. Margolis SA, Al-Marzouqi S, Revel T, Reed RL. Patient satisfaction with primary health care services in the United Arab Emirates. International journal for quality in health care. 2003;15(3):241-9.

23. Khoja TA, Al Shehri AM, Abdul Aziz AAF, Aziz K. Patterns of referral from health centres to hospitals in Riyadh region. EMHJ-Eastern Mediterranean Health Journal, 3 (2), 236243, 1997. 1997.

24. Mehrolhassani MH, Jafari Sirizi M, Poorhoseini SS, Yazdi Feyzabadi V. The challenges of implementing family physician and rural insurance policies in Kerman province, Iran: a qualitative study. Health and Development Journal. 2012;1(3):193-206.

25. Kavosi Z, Siavashi E. A Study of the Performance of Referral System in Urban Family Physician Program in Fars Province, Iran. Journal of Health Management \& Informatics. 2018;5(3):88-95

26. Tofighi S, Hamouzadeh P, Sadeghifar J, Raad Abadi M, Roshani M, Salimi M, et al. The compliance status of HACCP implementation requirements in nutrition departments of the selected hospitals of Tehran University of Medical Sciences. Jundishapur Journal of Health Sciences. 2012;4(3):15-23.

27. Akinci $F$, Mollahaliloğlu $S$, Gürsöz H, Öğücü $F$. Assessment of the Turkish health care system reforms: A stakeholder analysis. Health policy. 2012;107(1):21-30.

28. Nasrollahpour Shirvani $\mathrm{PhD}$ S. The implementation of family physician program in IR Iran: achievements and challenges. Journal of Babol University of Medical Sciences. 2014;16(3):15-26

29. Joolaee H, Alizadeh M, Fallahzadeh MH, Baseri A, Sayad M. Evaluation of clinical skills of family physicians in Fars province by means of observed structured clinical evaluation. Strides in Development of Medical Education. 2011;7(2):92-8.

30. Weist K, Högberg LD. ECDC publishes 2015 surveillance data on antimicrobial resistance and antimicrobial consumption in Europe. Eurosurveillance. 2016;21(46).

31. Ahmadi B, Arab M, Narimisa P, Janani L, Najafpoor G. Study of patterns medication prescribed by family physicians and per capita in the Ahvaz city. Journal of Healthcare Management. 2012(3):4.

32. Majdzadeh R. Family physician implementation and preventive medicine; opportunities and challenges. International journal of preventive medicine. 2012;3(10):665.

33. Olesen SW, Barnett ML, MacFadden DR, Lipsitch M, Grad $\mathrm{YH}$. Trends in outpatient antibiotic use and prescribing practice among US older adults, 2011-15: observational study. Bmj. 2018;362:k3155.
34. Azadbakht M, Mirjani SM, Yousofi M, Amini M. Drugs Prescription and Consumption in Mazandaran Province. Journal of Mazandaran University of Medical Sciences. 2015;24(122):44-52.

35. Alikhani A, Shahamat M, Ghaffarian Shirazi H. Survey on Antibiotic Prescription for under 14 Years Old Outpatient Children in General Practitioner Prescriptions in Yasuj. Armaghane danesh. 2006;10(4):83-91.

36. AHFS Pharmacologic-Therapeutic Classification. 2020.

37. Khadivi R, Yarahmadi A. The Drug Prescription Patterns and Utilization after Family Physician Program Implementation in Rural Health Centers of Isfahan District, Iran. Journal of Isfahan Medical School. 2014;31(271).

38. Guidelines for the Family Physician Program and Referral System in Urban Areas, Version 02 (2012).

39. Ofori-Asenso R, Brhlikova P, Pollock AM. Prescribing indicators at primary health care centers within the WHO African region: a systematic analysis (1995-2015). BMC Public Health. 2016;16(1):724.

40. Organization $\mathrm{WH}$. Using indicators to measure country pharmaceutical situations: fact book on WHO level I and level II monitoring indicators. Using indicators to measure country pharmaceutical situations: fact book on WHO level I and level II monitoring indicators2006.

41. Masoud A, Hekmat SN, Dehnavieh R, Haj-Akbari N Poursheikhali A, Abdi Z. An investigation of prescription indicators and trends among general practitioners and specialists from 2005 to 2015 in Kerman, Iran. International Journal of Health Policy and Management. 2018;7(9):818.

42. Yoon YK, Park GC, An H, Chun BC, Sohn J-W, KIM MJ, editors. 158trends of antibiotic consumption in Korea according to nation-wide reimbursement data (2008-2012): A population-based epidemiologic study. Open Forum Infectious Diseases; 2014: Oxford University Press.

43. Abbasian $\mathrm{H}$, Hajimolaali $\mathrm{M}$, Yektadoost $\mathrm{A}$, Zartab $\mathrm{S}$ Antibiotic utilization in Iran 2000-2016: Pattern analysis and benchmarking with organization for economic co-operation and development Countries. Journal of Research in Pharmacy Practice. 2019;8(3):162.

44. Barari M, Afrasiabian S, Mohsenpour B, Hajibagheri K, Sheikhesmaili F. Evaluation of appropriate antibiotic administration by general practitioners at the emergency department of a teaching hospital in Sanandaj, Iran. Scientific Journal of Kurdistan University of Medical Sciences. 2018;23(3):67-72.

45. Prah J, Kizzie-Hayford J, Walker E, Ampofo-Asiama A. Antibiotic prescription pattern in a Ghanaian primary health care facility. Pan African Medical Journal. 2017;28(1).

46. Sisay M, Mengistu G, Molla B, Amare F, Gabriel T. Evaluation of rational drug use based on World Health Organization core drug use indicators in selected public hospitals of eastern Ethiopia: a cross sectional study. BMC health services research. 2017;17(1):161.

47. Kashavarz A, Haji Mahmoudi H. Compare of health Costs in the provinces. Tehran. Ministry of Cooperatives, Labour and social welfare; 2014. 